\title{
Study of the Effects of Micro- and Nanolayered Structures on Mechanical Response of Microalloyed Steels
}

\author{
Janusz Majta ${ }^{*}$, Krzysztof Muszka, Lukasz Madej, Marcin Kwiecien, Paulina Graca \\ Faculty of Metals Engineering and Industrial Computer Science, AGH University of Science and Technology, Poland
}

Copyright (C) 2015 by authors, all rights reserved. Authors agree that this article remains permanently open access under the terms of the Creative Commons Attribution License 4.0 International License

\begin{abstract}
The specimens of microalloyed ferrite and austenite were severally deformed by complex metal forming processing (accumulative angular drawing - AAD, wire drawing - WD, wire flattening - WF) up to a total effective strain of 2.86, at room temperature. Electron backscattered diffraction (EBSD) and digital microstructure representation (DMR) analysis have been used to compare the deformed microstructural evolution of both model materials. The high angle boundaries associated with the deformation bands were formed at the total accumulated strains of $1.8 \div 2.0$ observed in the last passes of wire drawing. Then, ultrafine-grained and lamella structures were observed as a result of wire flattening. It was observed that the microalloyed ferrite refined more rapidly with work hardening than the microalloyed austenite. Evaluation of the processing route along with dislocation substructure co-operative relationship allows for design of ultrafine-grained and multilayered materials with controlled structure inhomogeneity in order to achieve desirable mechanical response.
\end{abstract}

Keywords Microalloyed Steel, Computer Modeling, Precipitation Strengthening, Work Hardening, Severe Plastic Deformation, Wire Drawing, Ultrafine-grained Materials

\section{Introduction}

Advanced materials have always been the object of intensive research. In the last few decades, a great number of metallic structural materials have been investigated by scientists from multidisciplinary fields. The key player between structural metallic materials is still steel. Hence, it is a natural consequence that advanced structural materials, as for example compositionally graded, ultrafine-grained (UFG) or multilayer structured steels are in the centre of interest of scientists. Also it can be stated that refinement of the microstructure is one of the main routes to improve the properties of metals and alloys. It is already verified, that any reduction in the grain size leads to significant enhancements in strength, toughness and fatigue resistance. Thus, the area of ultra-fine-scale structures does indeed appear to represent a new and rich area in which material science and engineering can develop new applications and properties by the exploitation of new production routes. Deformed or co-deformed multiphase materials, as for example multiphase or microalloyed steels offer an enormous potential for advanced alloy design. These advanced materials today represent the largest class of ultrahigh strength nanostructured bulk materials. However, industrial wide scale application of UFG materials remains limited, mainly because of their weak ductility due to a lack of work hardening. Considerable progress has been made during recent years towards understanding of the deformation and strengthening mechanisms that govern the mechanical response of UFG materials and some guidelines to improve their ductility have been proposed $[1,2]$. It has been found that one of the solutions would be to create inhomogeneous bimodal/multimodal microstructures where the ultrafine/nano grains would be responsible for providing the strength and coarse grains would be responsible for providing the work hardening and, thus, keep the reasonable level of ductility. Extensive research in the area of proposing a strong and yet ductile UFG or nanomaterial that may be produced in the continuous manner resulted in a new group of the products where the microstructural features are organized in clusters or layers [3]. These materials may be produced using a combination of severe plastic deformation (SPD) and advanced thermomechanical processing (ATP) methods. For example, recently developed accumulative angular drawing (AAD) [4-7] combined with wire drawing (WD) and wire flattening (WF) processes may be employed and effectively used to induce SPD effects in microalloyed steel wires. The AAD is characterized by a complex strain path history resulting from various deformation modes. Its main purpose is to produce wires that represent increased strength and ductility due to controlled deformation inhomogeneity $[4,8]$.

The present work introduces a systematic study of different factors affecting mechanical response of ultrafine-grained and multilayered microalloyed steel strengthened by fine precipitates. Presented in this paper results are in line with the present trends in materials engineering, namely because the research is focused on 
advanced materials and multi-scale modeling methods addressing both the knowledge of physically based multiscale deformation microstructure behaviors and better performance of structural metallic materials. The multiscale modeling is able to describe realistic multifunctional structures under various loading conditions. The novelty of the proposed ideas is to achieve mentioned above features in two different types of metallic system i.e. microalloyed ferrite (bcc) and a model microalloyed $\mathrm{Fe}-30 \mathrm{wt} \% \mathrm{Ni}$ austenite (fcc). Each of these materials represents different crystallographic structure and stacking fault energy (SFE) levels. It is already proved in the earlier works [9-12], that in the case of UFG microalloyed steels much stronger refinement, together with precipitation and solid solution strengthening can be obtained, what make these steels more attractive from the point of view of optimization of mechanical properties. Additionally, presented in this work results could be applied in a wide field of applications, using instead of layers compaction methods (deposition, laser beam melting, compacting, sintering etc.) typical deformation techniques (wire drawing, wire flattening and rolling).

\section{Experimental Procedure}

In order to assess the interrelationships between processing-microstructure-mechanical behaviors of UFG layered microstructure, two microalloyed steels wire rods (diameter of $6.5 \mathrm{~mm}$ ) with basic chemical composition shown in Table 1 were used in the current work. The wire rods were supplied with recrystallized polygonal ferrite and austenite microstructures and the mean grain size of $15 \mu \mathrm{m}$ (Fig. 1a) and $40 \mu \mathrm{m}$ (Fig. 1c), respectively (grain size was measured using mean linear intercept method). Additionally, the effects of precipitation occurring in the wire rods are presented for microalloyed ferrite (Fig 1b) and microalloyed autenite (Fig 1d). Both, the mean grain size and precipitation size were measured by automatic measuring system installed in microscope software (FEGSEM).

The production process of UFG layered material was carried out according to the general sequence as follows.
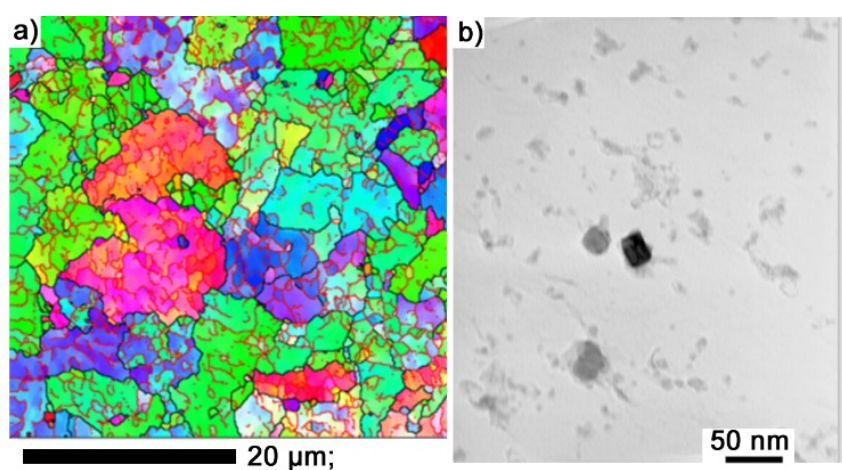

Figure 1. EBSD results showing maps of grain boundaries at the cross section of the studied materials - a), c) and precipitations $(\mathrm{TiC}, \mathrm{Nb}(\mathrm{C}, \mathrm{N}),(\mathrm{Ti}$,

$\mathrm{Nb}) \mathrm{CN}$ ) - b), d) observed in the investigated materials. Microalloyed ferrite - a), b; microalloyed austenite - c), d).
First, AAD process was performed to introduce severely deformed and inhomogeneous microstructures in the drawn wires. In this process, three deformation passes were applied and final wire diameter after AAD was $4.0 \mathrm{~mm}$. Total strains of 0.45 and 0.38 were applied in first and second pass, respectively, followed by the calibration pass with the strain of 0.14. Although, the AAD design allows for various combinations of die positioning to be used $[4,6]$, the present study was concentrated on the stepped die positioning, in which the offset from the drawing line between successive dies was equal to $10^{\circ}$. Additionally, after first pass, the wire was rotated by $180^{\circ}$ to obtain axi-symmetrical distribution of strain accumulation at the cross-section of a final product. Next, a multi-pass linear wire drawing (WD) process was carried out until wire diameter was reduced down to $1.96 \mathrm{~mm}$. For the comparison to the AAD process, a typical multi-pass wire drawing was conducted to reduce the wire diameter from 6.5 to $1.96 \mathrm{~mm}$. Finally, produced in such way wires were additionally deformed by wire flattening (WF) with equivalent strain of 0.45 to the thickness of $400 \mu \mathrm{m}$, so that the total strain accumulated in all processes was $\varepsilon=2.86$. Materials produced in all above-mentioned deformation schedules were subjected to microstructure analysis and mechanical testing. The changes in the microstructure were observed using optical ZEISS Axio Imager M1m microscope. Additionally, texture measurements were performed using EBSD/SEM technique. The EBSD data were collected using a FEI field emission gun scanning electron microscope (FEGSEM) equipped with a EDAX EBSD camera. TSL software was used for data acquisition while HKL Channel 5 software package was used for analysis. Assessment of precipitation behavior was performed using carbon extraction replicas method on FEI Tecnai 20 TEM equipped with EDAX EDX analyzer. Mechanical testing was performed using Instron 4502 tensile testing frame with the capacity of $10 \mathrm{kN}$. Finally, microhardness measurements have been performed in order to assess the mechanical inhomogeneity on the cross sections of produced flattened wires. Microhardness measurement were taken using Wolpert-Wilson TUKON 2500 micorhardness tester for Knoop method with $100 \mathrm{~g}$ load.

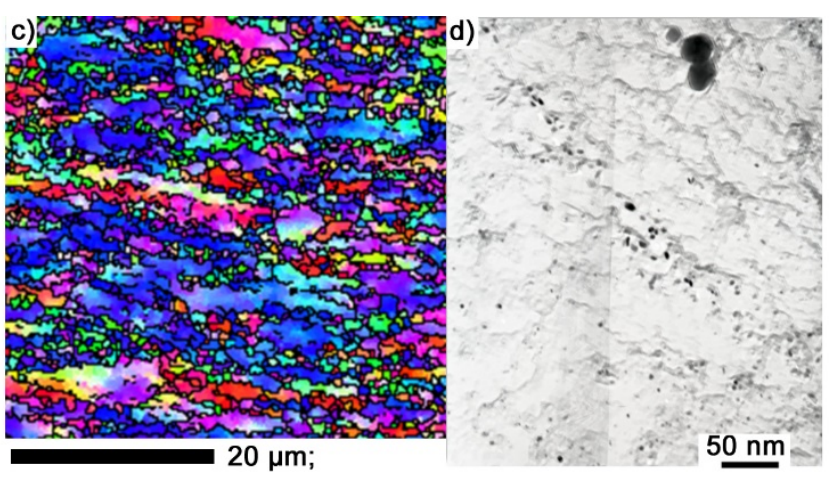


Table 1. Chemical composition of the investigated materials.

\begin{tabular}{|c|c|c|c|c|c|c|c|c|c|c|}
\hline Material & $\mathrm{C}$ & $\mathrm{Si}$ & $\mathrm{Mn}$ & $\mathrm{Nb}$ & $\mathrm{Ti}$ & $\mathrm{Cu}$ & $\mathrm{N}$ & $\mathrm{B}$ & $\mathrm{Ni}$ & $\mathrm{Co}$ \\
\hline Microalloyed ferrite & 0,07 & 0,29 & 1,36 & 0,067 & 0,03 & 0,16 & 0,0098 & 0,002 & - & - \\
\hline $\begin{array}{c}\text { Microalloyed } \\
\text { austenite }\end{array}$ & 0,047 & 0,30 & 1,64 & 0,097 & - & - & 0,0042 & - & 30,8 & 0,022 \\
\hline
\end{tabular}
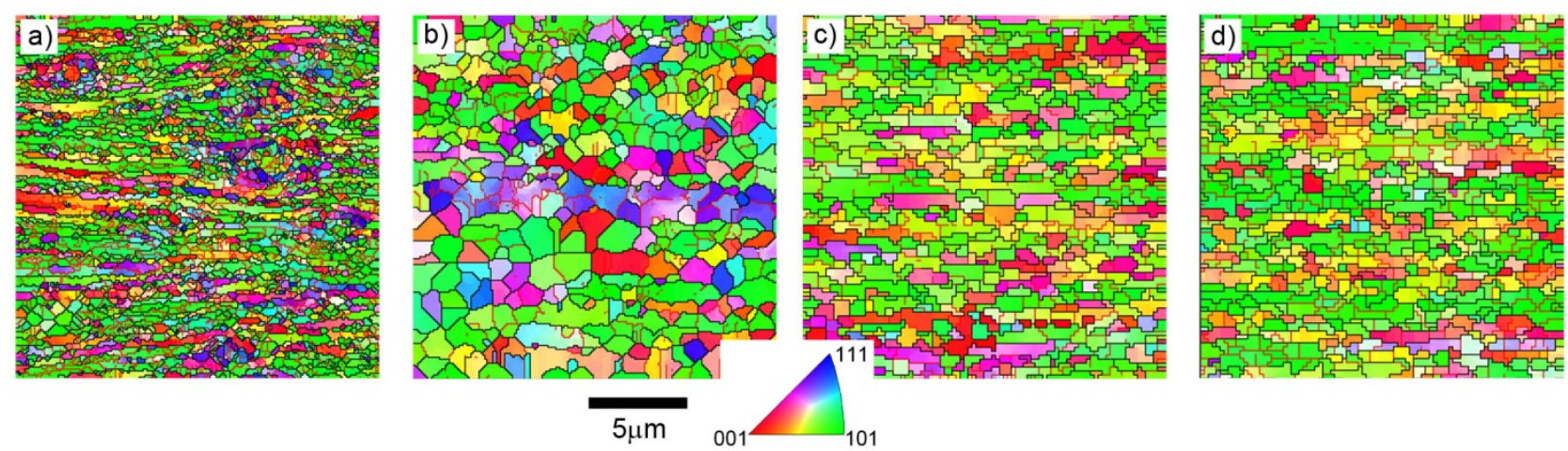

Figure 2. EBSD results showing maps of grain boundaries at the cross section of the studied material obtained after combined AAD $+\mathrm{WD}+\mathrm{WF}$ processing. Microstructures taken in the areas close to the surface a), c) and the centre b), d) of deformed material. Microalloyed ferrite - a), b; microalloyed austenite c), d).

\section{Experimental Results and Discussion}

Microstructures. The nanostructure metals and alloys produced by SPD are in extremely non-equilibrium state. Grains, subgrains, dislocation cells and precipitates frequently associated with dislocations are the most important microstructural features observed in these materials. In general, the grain structure of the UFG aggregate produced by SPD processing is characterized by presence of three grain types: i) dislocation free grains, ii) grains with dislocations (network and chaos), and iii) grains with dislocation cells and fragments [13]. However, depending on the crystallographic structure and SFE, the quantitative and qualitative descriptions of the structural components of polycrystal metals and alloys can be substantially different. Such situation is observed in the present study, where two different, from point of view of crystallographic structure (fcc, bcc) and SFE, materials were investigated i.e. microalloyed ferrite and microalloyed austenite. The present and previous results, for low carbon steel [6], confirmed that the strain path applied in the AAD process affects directly the microstructure and texture changes in the final product. The reason is that the strain accumulation in the drawn wires are direct results of combined effect of: reduction of the area, strain accumulation in the outer part of the wire due to bending/unbending process, and desired shear deformation. In the case of fcc metals, it is well known that the cell size evolves as a function of precipitate volume fraction [16]. The mechanism governing this behavior can be described by an Orowan-type formalism. A dislocation may avoid particles or obstacles by leaving the slip plane in the vicinity of each particle, or it may avoid the particles by the Orowan mechanism $[8,9,10]$. In the case of bcc metals, only the secondary walls spacing seems to depend on the precipitates.
It has been suggested, that the formation of cell is controlled mainly by the movements of primary screw dislocations, and this is mostly related to the lattice friction rather than to dislocation-dislocation interaction or dislocation-particle interactions [9]. The lattice friction is still important and seems to control the development of the planar dislocation substructure, which is thus independent on the volume fraction of precipitates; the closed cell structure results either from multiple slip activity or from interaction between dislocations and particles $[10,16]$. Hence, it can be expected that for both investigated in the present study microalloyed steels, effects of precipitation and solid solution strengthening, first of all $\mathrm{Nb}$ solute elements, indicatively influence the microstructure development. Also, deformation mechanisms, as a function of the grain size and accumulated deformation energy, significantly change in the microalloyed ferrite and austenite during the combined SPD processing. It is already proved, that when the grains are refined to the nano-size level, the main deformation goes along grain boundaries $[17,18]$. Hence, when the bimodal microstructure is produced, as it can be observed in the present study in the case of microalloyed ferrite (see Fig. 2 a,b), the deformation conditions can be improved due to the fact that two deformation mechanisms exist, i.e. in the grain boundaries and the grain interiors. Additionally, as it was already presented in [9] and also is shown in Fig. 2, the grain size, distribution and crystallographic orientations are clearly different in the cross section of the flattened wires. Mean grain size of microalloyed ferrite, in the centre of specimen. was about $1,2 \mu \mathrm{m}$ while in the areas close to the surface of about $0,1 \mu \mathrm{m}$. It is observed, that in the case of microalloyed ferrite, inhomogeneity of the microstructure development, first of all the progress of grain refinement, is the characteristic feature of the flattened wires.

It has been already proved that the grain refinement 
process during SPD is an effect of continuous dynamic recrystallization (CDR) (recrystallization in situ) where, in contrast to the typical dynamic recrystallization, new grain boundaries are continuously created from geometrically necessary dislocations [19]. This process is directly connected to the SFE of the material. In general, in bcc metals high SFE favors CDR processes [8]. In the case of microalloyed austenite, with low SFE, the situation is quite different. We should remember that investigated in the present study fcc alloy (room temperature microalloyed austenite) is in non-equilibrium state (deformation-induced martensite transformation in the austenite occurs) and the twinning is the major deformation mode, as usual in the low temperature condition of austenite, at least in the first stages of the deformation process (AAD+WD).

Such situation leads to more homogeneous microstructures of microalloyed austenite (see Fig. 2c, d), comparing to the microalloyed ferrite. It can be clearly seen, that in this case the mean grain size in the areas close to the surface and in the centre of specimens are respectively 365 $\mathrm{nm}$ and $389 \mathrm{~nm}$. However, presence of refined pearlite colonies as well as formation of the lamellar structure, especially in case of microalloyed austenite, was also clearly observed in the investigated steels (Fig. 3). Additionally, low SFE of the microalloyed austenite, as well as significantly refined in the initial stages of deformation grains can be notable effective in retardation of the twinning mechanism. All of the mentioned above features can be involved in the microstructure homogenization process of microalloyed austenite. Hence, the conclusion can be drawn that bcc metals are better "indicators" of the deformation inhomogeneity in SPD processing than low SFE fcc metals.
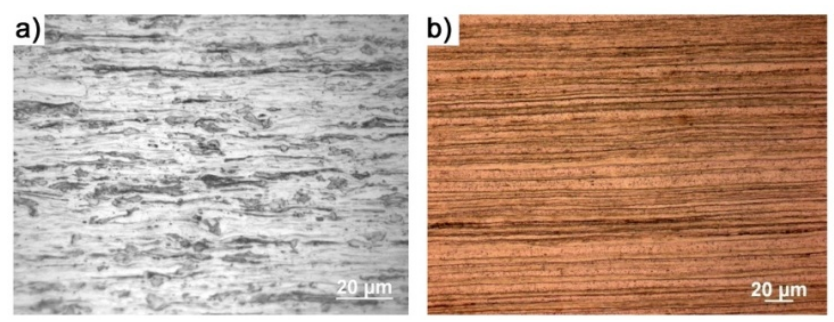

Figure 3. Lamellar structures observed in the investigated materials obtained after combined AAD $+\mathrm{WD}+\mathrm{WF}$ processing. Microalloyed ferrite a); microalloyed austenite - b).

Mechanical Properties. It is well recognized that high strength and good ductility are mutually exclusive, and improving both in the SPD processing is very difficult. Additionally, the mechanical response of the specimens produced, as it was in the present study, by combined SPD processing changes with the investigated materials (Fig. 4). It is confirmed that the strong grain refinement causes valuably increase in strength [20]. Also, the decrease in ductility can be easily observed. The maximum uniform elongation significantly decreases with grain refinement in both deformed materials and reaches value of 0.0149 and
0.0073 for microalloyed ferrite and microalloyed austenite, respectively (Fig. 4a). Hence, it is very interesting observation that the high strength ferritic steel represents better ductility than austenitic steel. The main reason for this enhancement of both mechanical properties in the case of microalloyed ferrite (bcc) is a combination of high flow stress and higher than in the austenitic steel (fcc) rate of strain hardening. However, the Considere criterion shows that the onset of necking in both microalloyed steels is effectively retarded due to presence of fine precipitates and solute atoms. It is also evident, that in the case of microalloyed ferrite, the combined (AAD + WD) deformation process is more beneficial in the improvement of mechanical properties than in the case of regular wire drawing. Fine precipitates present in both microalloyed steels are active in the trapping of dislocations. The larger precipitations pin only a limited number of dislocation walls. In the UFG materials, the hard precipitates also initiate, drag and pin dislocations, hence the dislocation structure rearrangement such as dynamic recovery is reduced. As a result, substantial dislocation storage is observed that in turn is required for compatible plastic strains, allowing a high strain-hardening rate that leads to larger uniform strains while increasing strength $[12,13]$. As the volume fraction of precipitates increases, the average size of closed cells decreases [14]. Also the spacing between cell walls becomes lower with increasing precipitates volume fraction. Additionally, when the solute atoms are segregating at the grain boundaries and the microstructure is significantly refined additional dislocations are generated from the grain boundaries what supports the deformation process. This effect leads to enhanced ductility of already strengthened by the grain refinement and the Orowan mechanism microalloyed steel.

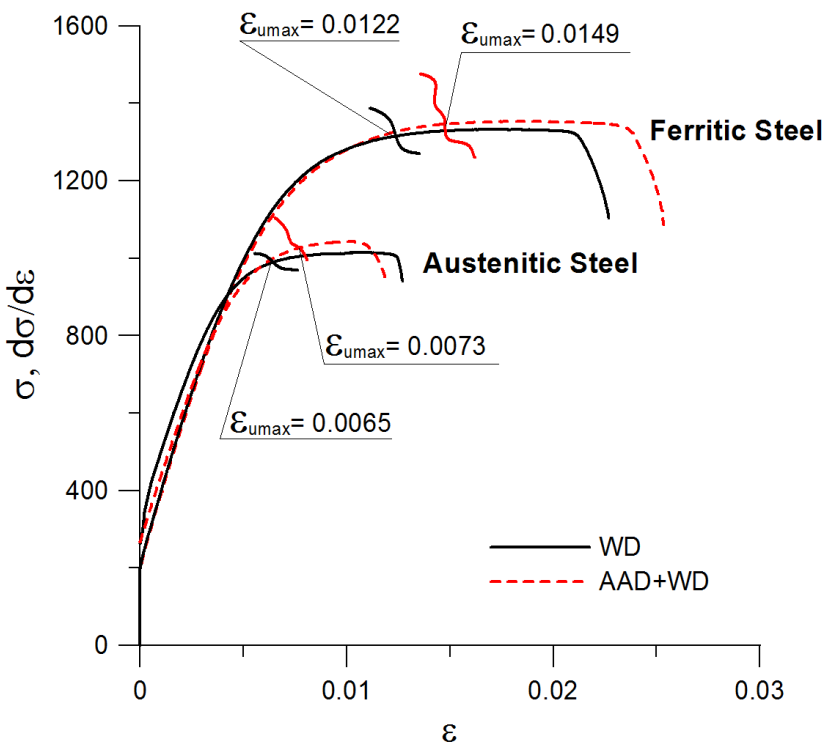

(a) 


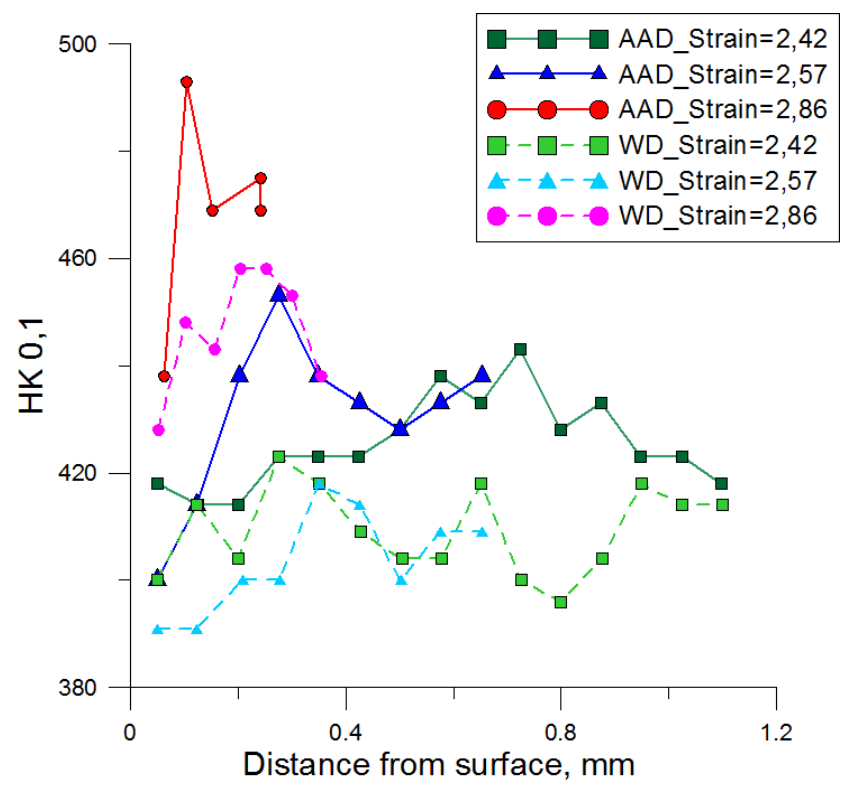

(b)

Figure 4. Stress-strain curves obtained under quasi-static tension tests of the specimens of microalloyed ferrite and austenite after $\mathrm{AAD}+\mathrm{WD}+\mathrm{WF}$ processing with marked uniform elongation values assessed using Considerè criterion - a). Microhardness measurements on the cross section of specimens of microalloyed ferrite after first (Strain $=2.42$ ), second $($ Strain $=2.57)$ and third $($ Strain $=2.86)$ stage of flattening $-b)$. WD - typical wire drawing.

Application of the complex deformation modes $(\mathrm{AAD}+\mathrm{WD})$, instead of typical wire drawing (WD), and proper control of the level of deformation and microstructural inhomogeneity results in the improvement of both strength and ductility (Fig. 4). Mechanical properties measured in the tensile tests clearly show that by applying the combined metal forming processing $(\mathrm{AAD}+\mathrm{WD}+\mathrm{WF})$ indicative enhancement of ductility (Fig. 4a) and strength (Fig. 4a,b) of the microalloyed steel wires can be obtained. Another advantage of UFG microstructure, produced first of all in the outer layers of microalloyed ferrite specimens, is enhanced fatigue properties due to the large number of grain boundaries, which impede crack propagation [21].

\section{Computer Modeling}

From a microscopic viewpoint, the plastic deformation and work hardening of a crystal are caused by dislocation motions and accumulation. In order to model mechanical behavior of UFG materials, there is a need, first of all, to understand the deformation and strengthening mechanisms governing the plastic deformation of such material. To capture these phenomena in a sufficient way, multiscale modeling approach needs to be utilized, since the conventional material models are not amendable to bridge the gap arising from different scales at which those phenomena are taking place [22]. Thus, hierarchical material models involving different scales are required in order to predict some of the interactions between microscopic dislocation fields and macroscopic deformation fields computationally. The most commonly accepted type of models of grain refinement due to SPD conditions are based on the statement that a dislocation cell structure forms in the beginning of deformation and then, because of continuously increased misorientation angle, low angle grain boundaries transform to the final refined grain structure. This model was employed in the present work by utilization of the Digital Material Representation (DMR) approach that allows to represent microstructure in the finite element based codes in an explicit manner [23]. The real microstructure evolution during SPD processing needs a more detailed representation of the dislocation population [24]. Hence, it is still valid, as it was mentioned elsewhere [25], that understanding the self-organization processes leading to the emergence of a dislocation cell structure is one of the greatest unsolved problems of the dislocation theory.

In the present study, additional emphasis was put on the proper representation of the effects of precipitation hardening that are characteristic for investigated here microalloyed steels. The idea of taking into account this strengthening mechanism is presented in Fig. 5. DMR unit cell was created as a two phase material i.e. steel matrix with dispersed fine particles of the $\mathrm{Nb}(\mathrm{C}, \mathrm{N})$ precipitates. The properties of the steel matrix were assigned to each grain separately using material data generated from Chaboche model [26] (slightly diversified according to Gauss function in order to take into account crystallographic orientation changes of the grains). The size of the $\mathrm{Nb}(\mathrm{C}, \mathrm{N})$ particles was chosen based on the graph presented in Fig. 5. Precipitation strengthening is a hardening mechanism that can be governed by two mechanisms - depending on the type and size of the particles [27]. In the first mechanism, dislocations moving during deformation can cut through the particles that are larger, softer and yet coherent with the matrix Mott-Nabarro mechanism. The finer and harder particles (e.g. strain-induced precipitates of $\mathrm{Nb}(\mathrm{C}, \mathrm{N}))$ that cannot be cut through moving dislocations work as obstacles for them so they can only by by-passed what causes much higher strengthening effect - Orowan mechanism. As it can be seen from the graph in Fig. 4 there is an optimal size of the second phase particles that causes maximum strengthening effect for $\mathrm{Nb}(\mathrm{C}, \mathrm{N})$ it is around $10-50 \mathrm{~nm}$. Such particles were observed also in both of the investigated microalloyed steels. In the present work, hard particles with this size range were created within the DMR unit cells. 


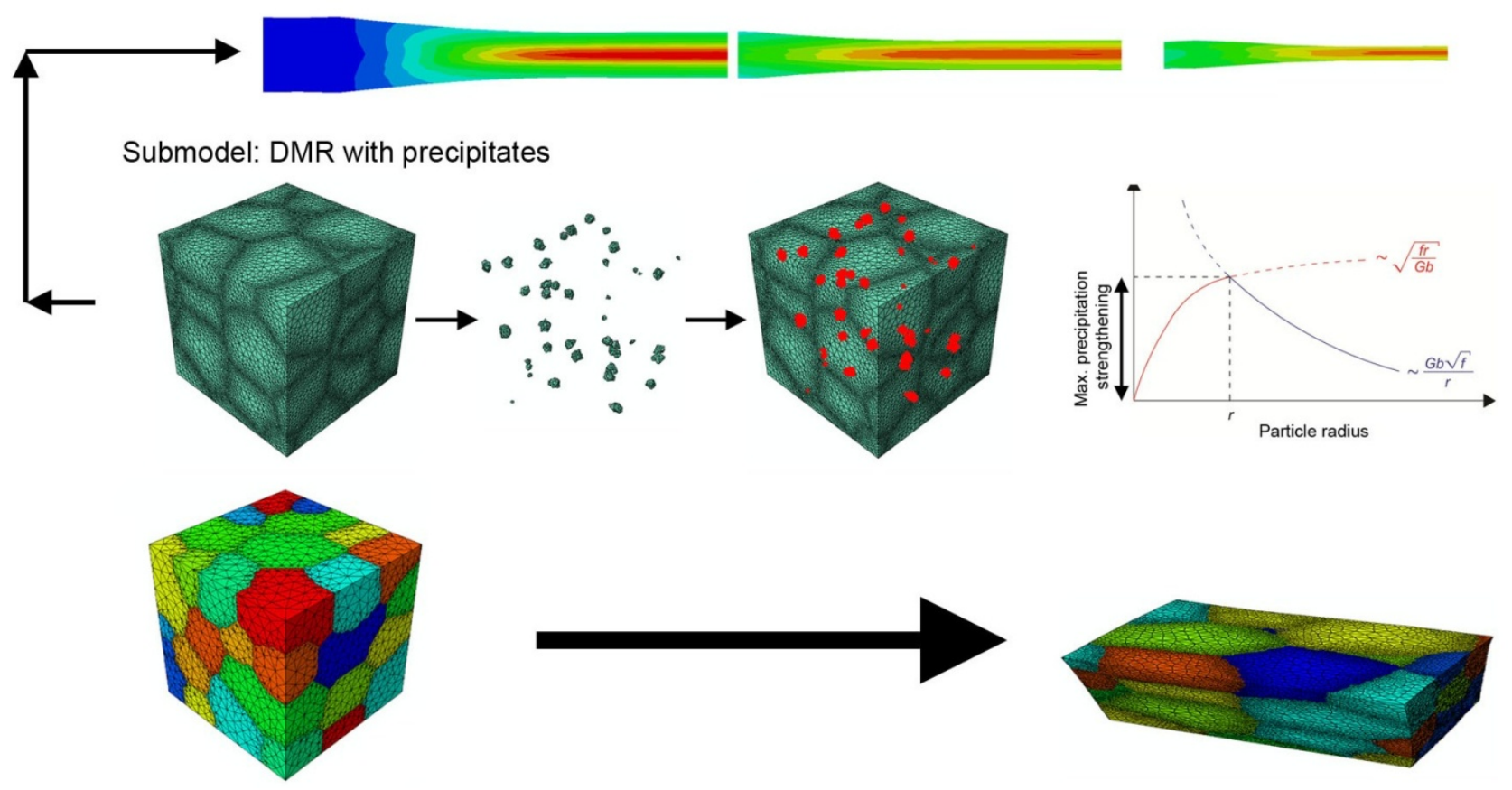

Figure 5. Way of taking into account the precipitation hardening effects in the current modeling work.
a) ferrite (bcc)
b) autenite (fcc)

c) DMR (fcc)

Initial wire diameter: $1.92 \mathrm{~mm}$

Wire Flattening:
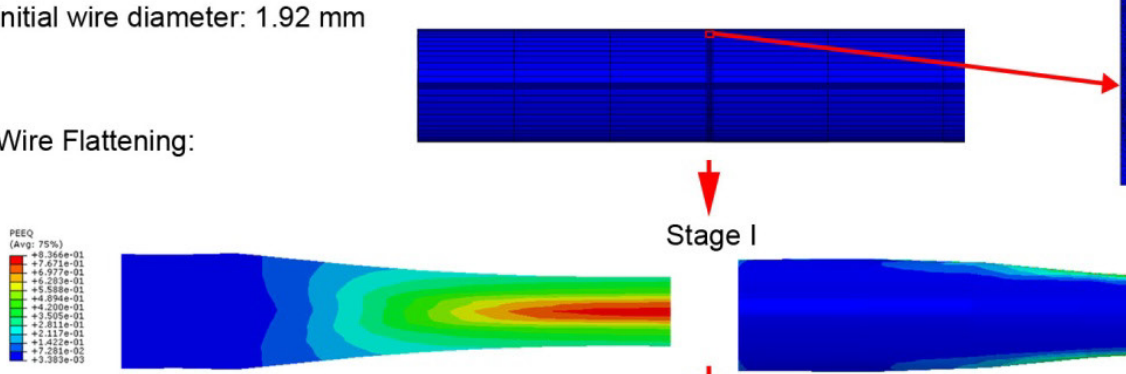

Stage I
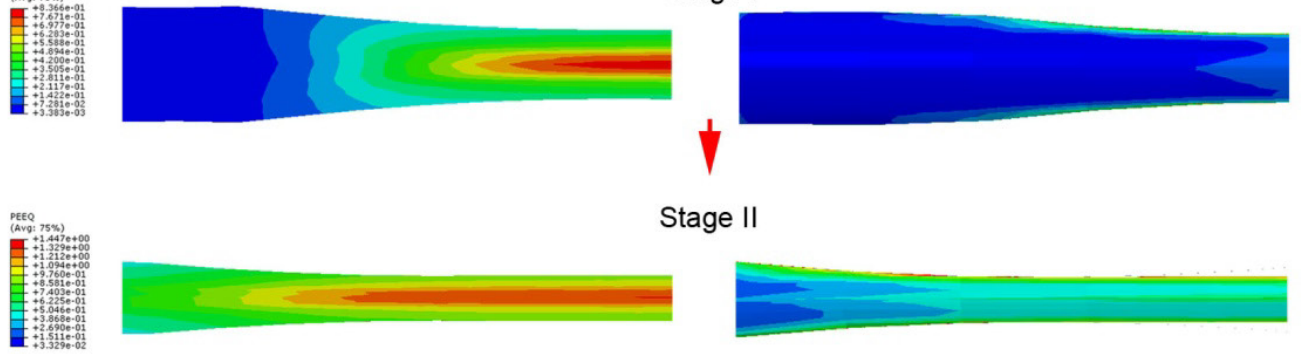

Stage II
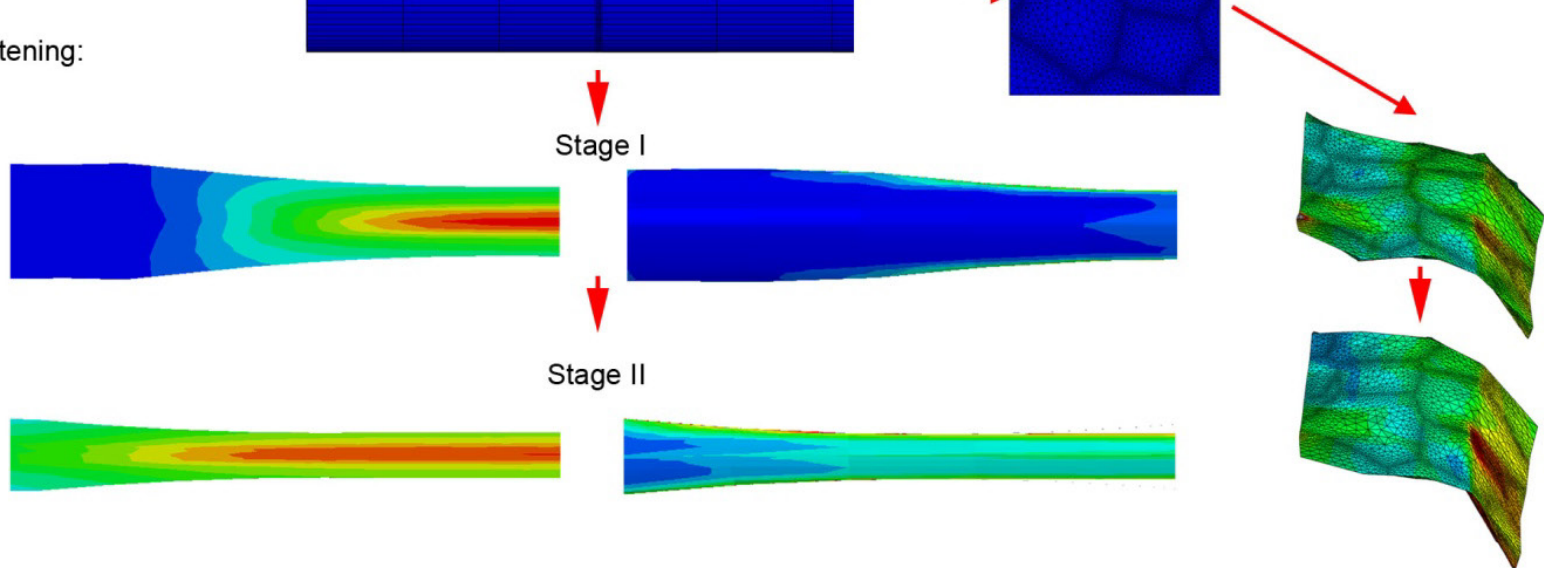

Figure 6. Examples of the numerical modeling results: equivalent plastic strain distribution in global model after 2 stages of wire flattening in ferrite -a) and austenite -b), and in DMR of austenite.

Fig. 6 shows initial modeling results of equivalent plastic strain distributions after first two steps of the wire flattening process using proposed multiscale modeling approach. First of all, it can be seen that developed in this work modeling strategy was able to capture differences in the levels of strain inhomogeneity between fcc and bcc structures at micro scale (Fig. 6a and b). Application of the multiscale approach combined with DMR allowed further improvement of the accuracy of the model. It can be seen from Fig. $6 \mathrm{c}$ that thanks to this approach, deformation gradient within the grains and in the grain boundaries areas can be seen. It also captures the grain shape changes including onset of lamellar structure development.

Based on these initial results, it can be summarized that the proposed model properly captures the effects of complex deformation history and shows potential in the prediction of the material deformation behavior in these complex deformation processes. Proposed approach seems to be very promising, as representation of the microstuctural features at various scales, offers much more physically-based numerical tool for the simulation of the UFG layered materials.

\section{Conclusions}

The discussed processing route allows for obtaining significant refinement of the grain size. The approach is typical for obtaining of UFG materials and eventually 
multilayered structures by top-down techniques. Again, it was proved, that in the UFG and lamella structures fine precipitate affects significantly the strength, according to the Orowan strengthening mechanism. The results show that, the presence of precipitates in the severally deformed and multilayered structures can be not only the key factor in strengthening process, but also critically supports the grain refining process, first of all in the bcc metals. It was clearly demonstrated that substantial improvement of mechanical properties and stabilization of nanocrystalline grains combined with obtaining of the layered structure can be achieved due to several, closely linked phenomena, such as solid solution and precipitation strengthening as well as presence of refined pearlite colonies. However, this effect is more pronounced in the case of microalloyed ferrite. It has been shown experimentally and supported by numerical multiscale analysis of microstructure evolution that combination of the different metal forming operations allows for design of the complex structures in microalloyed steels.

\section{Acknowledgements}

Research presented in the current work was performed with the financial support provided by National Science Centre Poland (grant no. DEC-2012/05/B/ST8/00215).

\section{REFERENCES}

[1] P.D. Hodgson and H. Beladi: Steel-Grips Vol. 2, Suppl. (2004), p.

[2] K. Muszka, J. Majta and P.D. Hodgson, in: MS\&T 2007 Conference and Exhibition, Detroit, (2007)

[3] A. Misra, J.P. Hirth and H. Kung: Phil. Mag. A Vol. 82 (2002), p. 2935

[4] J. Majta, J. Luksza and M. Wielgus, PL Patent P.388532 (2009)

[5] K. Muszka, L. Madej and J. Majta: Mater . Sci. Eng. A Vol. 574, (2013) p. 68

[6] J. Majta, K. Muszka, S. Dymek, D. Dziedzic and M. Kopyściański, in: Proceedings of the 14th International Conference on Metal Forming 2012, edited by J. Kusiak, J. Majta, D. Szeliga, Steel Research International, Spec. Ed., Krakow (2012).
[7] M. Wielgus, J. Majta, J. Łuksza and P. Packo: Steel Res. Int. Vol. 81 (2010), p.490

[8] J. Majta and K. Muszka, in: Microstructure evolution in metal forming processes, eds. J. Lin, D. Balint, M. Pietrzyk, chapter 9, WP Woodhead Publishing Limited, Cambridge (2012)

[9] Yang Xiang, D.J. Srolovitz, L.T. Cheng, E. Weinan: Acta Materialia 52 (2004) p. 1745

[10] F. J. Humphreys and J. W. Martin, J., Phil. Mag., 16, (1967) p. 927.

[11] K. Muszka, M. Wielgus, J. Majta, K. Doniec, and M. Stefańska-Kądziela, Mater. Sci. Forum. Vols. 654-656 (2010), p. 314

[12] M. Stefanska-Kadziela, J. Majta, S. Dymek, and K. Muszka: Arch Metall Mater Vo. 52 (2007) Vol. 52, p. 223

[13] A. Zurek, J. Majta, K. Muszka, and M. Wielgus, in: Proceedings of DYMAT 2009, Brussels, Belgium (2009), 2, p. 1015.

[14] E. Ma, JOM. Vol. 4 (2006), p. 49

[15] E.V. Kozlov, A.N. Zhdanov, N.A. Popova, E.E. Pekarskaya and N.A. Koneva: Mater. Sci. Eng. A Vols. 387-389 (2004), p.789

[16] G.Z. Voyiadjis, F.H. Abed: Arch. Mech. 57, 4 (2005) p. 299

[17] M.A. Meyers, A. Mishra and D.J. Benson, Prog. Mater. Sci. 51 (2006) p.427

[18] D.G. Morris, Rev. Metal. Madrid 46, 2, (2010) p. 173

[19] A.Oscarsson, B. Hutchinson, B. Nicol, P. Bate and H. E. Ekström, Mater Sci Forum, 157-162 (1994) p. 1271

[20] N. Hansen / Scripta Materialia 51 (2004) p. 801

[21] Z. Valiev and T.G. Langdon: Metall. Mater. Trans. A Vol. 42 (2011), p. 2942

[22] K. Muszka: Mater. Sci. Eng. A Vol. 559 (2013) p. 635

[23] L. Madej, L. Rauch, K. Perzynski and P. Cybulka: Arch. Civ. Mech. Eng. Vol. 11 (2011), p. 661

[24] D. Svyetlichnyy, J. Majta and K. Muszka: Steel Res. Int. Vol. 79 (2008), p. 452

[25] Y. Estrin and A. Vinogradov: Acta Mater. Vol. 61 (2013), p. 782

[26] J. Lemaitre and J. Chaboche: Mechanic of solid materials (Cambridge University Press, UK 2000)

[27] B.C. De Cooman, J.G. Speer, I.Y. Pyshmintsev and N. Yoshinaga, in: Materials Design: The key to modern steel Products, Grips Media GmbH (2007). 